\title{
ON THE COEFFICIENTS OF UNIVALENT FUNCTIONS ${ }^{1}$
}

\section{ZEEV NEHARI}

Let $S$ denote the family of regular univalent functions

$$
f(z)=z+a_{2} z^{2}+a_{3} z^{3}+\cdots
$$

in the unit disk, and set

$$
A_{n}=\sup \left|a_{n}\right|, \quad f(z) \in S .
$$

While Bieberbach's conjecture that $A_{n}=n$ has to date been verified only up to $n=4$ (the case $n=4$ only very recently and by an extraordinary analytical effort [1]), even the much weaker "asymptotic Bieberbach conjecture"

$$
\limsup _{n \rightarrow \infty} \frac{A_{n}}{n}=1
$$

has so far been neither proved nor disproved.

Littlewood [2] has suggested the conjecture

$$
\left|a_{n}\right| \leqq 4|d| n, \quad f(z) \neq d(|z|<1) .
$$

Because of $|d| \geqq 1 / 4,(4)$ is weaker than the Bieberbach conjecture but, as shown by the function $f(z)=z(1-z)^{-2}=z+2 z^{2}+3 z^{3}+\cdots$ $(f(z) \neq-1 / 4)$, it would still be sharp. In the present note we shall show that the truth of Littlewood's conjecture (4) would follow from the proof of the asymptotic result (3). More specifically, we shall prove the following theorem. then

If (1) is a function of $S$ and $d$ is a value such that $f(z) \neq d$ in $|z|<1$,

$$
\left|a_{n}\right| \leqq 4|d| \alpha n
$$

where

$$
\alpha=\limsup _{n \rightarrow \infty} \frac{A_{n}}{n}
$$

and $A_{n}$ is defined by (2).

The proof is based on the following

Received by the editors April 16, 1956.

1 This research was supported by the United States Air Force Office of Scientific Research. 
Lemma. If

$$
F(z)=b_{1} z+b_{2} z^{2}+\cdots
$$

is regular and univalent, and $F(z) \neq 1$, in $|z|<1$, then the same is true of the function

$$
F_{1}(z)=b_{1}^{(1)} z+b_{2}^{(2)} z^{2}+\cdots=2 F\left(z^{2}\right)-2\left\{F\left(z^{2}\right)\left[F\left(z^{2}\right)-1\right]\right\}^{1 / 2} .
$$

The lemma is easily verified. If $\zeta$ and $\eta$ are two values in the unit disk such that $F_{1}(\zeta)=F_{1}(\eta)$, an elementary manipulation shows that we must also have $F\left(\zeta^{2}\right)=F\left(\eta^{2}\right)$ and therefore, because of the univalence of $F(z), \zeta^{2}=\eta^{2}$. But $F_{1}(\zeta)=F_{1}(-\zeta)(\zeta \neq 0)$ is excluded because of $F_{1}(\zeta)-F_{1}(-\zeta)=-2\left(F\left(\zeta^{2}\right)\left[F\left(\zeta^{2}\right)-1\right]\right)^{1 / 2}$. Since, in view of $F(0)=0$ and $F(z) \neq 1, F_{1}(z)$ is single-valued in $|z|<1$, it is thus found to be univalent. That $F_{1}(z)$ does not take the value 1 is a consequence of $F(z) \neq \infty$ and the fact that $w-(w(w-1))^{1 / 2} \neq 1 / 2$ for finite $w$. This proves the lemma.

Clearly, the procedure leading from $F(z)$ to $F_{1}(z)$ may be iterated, and we thus obtain a sequence of univalent functions $F_{1}(z), F_{2}(z), \cdots$ such that

$$
F_{\nu+1}(z)=2 F_{\nu}\left(z^{2}\right)-2\left\{F_{\nu}\left(z^{2}\right)\left[F_{\nu}\left(z^{2}\right)-1\right]\right\}^{1 / 2} .
$$

If $F_{\nu}(z)=b_{1}^{(\nu)} z+b_{2}^{(\nu)} z^{2}+\cdots$ is the power series expansion of $F_{\nu}(z)$, (8) shows that

$$
b_{2 k}^{(\nu)}=2 b_{k}^{(\nu-1)}, \quad k=1,2, \cdots,
$$

and $\left|b_{1}^{(\nu)}\right|=2\left(\left|b_{1}^{(\nu-1)}\right|\right)^{1 / 2}$, or

$$
\frac{1}{4}\left|b_{1}^{(\nu)}\right|=\left(\frac{1}{4}\left|b_{1}^{(\nu-1)}\right|\right)^{1 / 2} \text {. }
$$

Let now $f(z)$ be a function of $S$ and let $d$ be such that $f(z) \neq d$ in $|z|<1$. We choose a fixed $n$ and apply the lemma successively to the function $F(z)=d^{-1} f(z)$ and to the function $F_{1}(z), \cdots, F_{\nu}(z)$ obtained from it by means of (7) and (8). If we set $m=2^{v} n$ and apply (9), we find that the $m$ th coefficient of the resulting univalent function is $b_{m}^{(\nu)}=2^{\nu} b_{n}$. The function $f_{\nu}(z)=\left[b_{1}^{(\nu)}\right]^{-1} F_{\nu}(z)$ is in $S$, and it thus follows from (2) that $\left|b_{m}^{(\nu)}\right| \leqq A_{m}\left|b_{1}^{(\nu)}\right|$. In view of (10), and the fact that $|d| \geqq 1 / 4$, we have

$$
\left|b_{1}^{(\nu)}\right|=4\left(\frac{\left|b_{1}\right|}{4}\right)^{2^{-\nu}}=4\left(\frac{1}{4|d|}\right)^{2^{-\nu}} \leqq 4
$$


Hence,

$$
\begin{aligned}
\frac{\left|a_{n}\right|}{n} & =\frac{\left|b_{n}\right||d|}{n}=\frac{\left|b_{m}^{(p)}\right||d|}{2^{p} n}=\frac{\left|b_{m}^{(p)}\right||d|}{m} \\
& \leqq \frac{A_{m}}{m}|d|\left|b_{1}^{(p)}\right| \leqq 4|d| \frac{A_{m}}{m} .
\end{aligned}
$$

If we let $\nu \rightarrow \infty, m$ will tend to $\infty$ through the sequence $\left\{2^{\prime \prime} n\right\}$, and we may therefore conclude from (6) that

$$
\limsup _{\nu \rightarrow \infty} \frac{A_{m}}{m} \leqq \alpha .
$$

It follows that $\left|a_{n}\right|$ is bounded by the right-hand side of (5), and the proof is complete.

We finally remark that the function $f_{\nu}(z)$ leaves out the value $\left[b_{1}^{(\nu)}\right]^{-1}$. Since, for sufficiently large $\nu$, the quantity

$$
\left|b_{1}^{(v)}\right|^{-1}=\frac{1}{4}(4|d|)^{2^{-\nu}}
$$

is arbitrarily close to $1 / 4$, the theorem will remain true if in the definition (2), (6) of $\alpha$ we replace $S$ by the sub-class of functions which leave out a value $d$ such that $1 / 4 \leqq|d| \leqq 1 / 4+\epsilon(\epsilon>0)$, where $\epsilon$ may be taken arbitrarily small.

\section{REFERENCES}

1. P. Garabedian and M. Schiffer, Proof of the Bieberbach conjecture for $n=4$, Journal of Rational Mechanics and Analysis vol. 4 (1955) pp. 427-465.

2. J. E. Littlewood, On inequalities in the theory of functions, Proc. London Math. Soc. (2) vol. 23 (1925) pp. 481-519.

Carnegie Institute of Technology 